International Journal of Computer Networks \& Communications (IJCNC) Vol.5, No.1, January 2013

\title{
FurTher RESUlts ON THE DiRAC DELTA APPROXIMATION AND THE MOMENT GENERATING FUNCTION TECHNIQUES FOR ERror Probability ANALYSis IN FADING CHANNELS
}

\author{
Annamalai Annamalai ${ }^{1}$, Eyidayo Adebola ${ }^{2}$ and Oluwatobi Olabiyi ${ }^{3}$ \\ Center of Excellence for Communication Systems Technology Research \\ Department of Electrical \& Computer Engineering, \\ Prairie View A\&M University, Texas \\ 1 aaannamalai@pvamu.edu, 2eyidayoadebola@yahoo.com, \\ 3 engr3osegmail.com
}

\begin{abstract}
In this article, we employ two distinct methods to derive simple closed-form approximations for the statistical expectations of the positive integer powers of Gaussian probability integral $E_{\gamma}\left[Q^{p}(\sqrt{\beta \Omega \gamma})\right]$ with respect to its fading signal-to-noise ratio (SNR) $\gamma$ random variable. In the first approach, we utilize the shifting property of Dirac delta function on three tight bounds/approximations for $Q($.$) to circumvent the$ need for integration. In the second method, tight exponential-type approximations for $Q($.) are exploited to simplify the resulting integral in terms of only the weighted sum of moment generating function (MGF) of $\gamma$. These results are of significant interest in the development of analytically tractable and simple closedform approximations for the average bit/symbol/block error rate performance metrics of digital communications over fading channels. Numerical results reveal that the approximations based on the MGF method are much more versatile and can achieve better accuracy compared to the approximations derived via the asymptotic Dirac delta technique for a wide range of digital modulations schemes and wireless fading environments.
\end{abstract}

\section{KEYWORDS}

Moment generating function method, Dirac delta approximation, Gaussian quadrature approximation.

\section{INTRODUCTION}

The Gaussian $Q$-function is defined as

$$
Q(x)=\frac{1}{2} \operatorname{erfc}\left(\frac{x}{\sqrt{2}}\right)=\int_{x}^{\infty} \frac{1}{\sqrt{2 \pi}} \exp \left(-y^{2} / 2\right) d y, x \geq 0
$$

which corresponds to the complement of the cumulative distribution function (CDF) of a normalized (zero-mean, unit variance) Gaussian random variable. This mathematical function plays a vital role in the analysis and design of digital communications since the conditional error probability (CEP) of a broad class of coherent modulation schemes can be expressed either in terms of $Q(x)$ alone or as a weighted sum of its integer powers (e.g., see Table 1, [1, Eqs. (8.36)(8.39)], [2, Chapter 4]). In addition, system performance measures such as the average symbol, bit or block error probabilities in fading channels typically involve taking the statistical expectation of $Q(x)$ and its integer powers with respect to the random variable that characterizes 
the fading channel (i.e., $\left.E\left[Q^{p}(x)\right]\right)$. However, analytical difficulties associated with evaluating $E\left[Q^{p}(x)\right]$ from its canonical integral representation of (1) (owing to the presence of the argument of the function in the lower limit of the integral) have led to the development of alternative exponential-type integral representations for the $Q$-function and its integer powers [1, Eqs. (4.2), (4.9), (4.31) and (4.32)], analytically simple and tight closed-form bounds and approximations for $Q(x)$ [3]-[16], characteristic function method [21] and the asymptotic analysis approach [17]-[19].

Table 1. CEP of several coherent digital modulation schemes.

\begin{tabular}{|l|l|}
\hline Modulation & Conditional Symbol Error Probability $\boldsymbol{P}_{s}(\gamma)$ \\
\hline BPSK & $Q(\sqrt{2 \Omega \gamma})$ \\
\hline$M$-PSK & $2 Q(\sqrt{2 \Omega \gamma} \sin (\pi / M)), M \geq 4$ (approx.) \\
\hline$M$-QAM & $\begin{array}{l}4 \lambda Q(\sqrt{3 \Omega \gamma /(M-1)})-4 \lambda^{2} Q^{2}(\sqrt{3 \Omega \gamma /(M-1)}), \\
\text { where } \lambda=(\sqrt{M}-1) / M\end{array}$ \\
\hline $\begin{array}{l}\text { Differentially Encoded BPSK } \\
\text { (DE-BPSK) }\end{array}$ & $2 Q(\sqrt{2 \Omega \gamma})-2 Q^{2}(\sqrt{2 \Omega \gamma})$ \\
\hline DE-QPSK & $4 Q(\sqrt{\Omega \gamma})-8 Q^{2}(\sqrt{\Omega \gamma})+8 Q^{3}(\sqrt{\Omega \gamma})-4 Q^{4}(\sqrt{\Omega \gamma})$ \\
\hline
\end{tabular}

Note: $\Omega=E_{s} / N_{o}$ denotes the received signal-to-noise ratio (SNR).

But the appeal of a single exponential-type integral representation (with finite integration limits) for the $Q$-function is restricted to the first four powers of $Q(x)$. Also, majority of existing bounds and approximations (with the exception of [4], [8], [12]-[14]) are not in the "desirable exponential form" for facilitating the task of statistical averaging of the CEP in generalized multipath and multichannel fading environments. Moreover, only [5], [6], [9], [11] and [15] have considered the problem of finding $E\left[Q^{p}(x)\right]$ in closed-form, and subsequently applied their results for average symbol error rate (ASER) analysis of differentially-encoded binary phase shift keying (DE-BPSK) and quaternary phase shift keying (DE-QPSK) digital modulation schemes in Nakagami- $m$ fading. Although [6] may be extended to other fading environments, their final expression will involve the computation of higher-order derivatives of the MGF of SNR. It was also pointed out in [11] that the accuracy of [6, Eqs. (10)-(11)] deteriorates considerably with the increasing value of Nakagami- $m$ fading severity index. While [11, Eq. (10)] (which utilizes a semi-infinite Gauss-Hermite quadrature approximation for $Q(x)$ ) can achieve better accuracy compared to [5], [6] and [9], its solution is limited to only Nakagami- $m$ fading. In [16], Jang suggested using an asymptotic Dirac delta approximation $Q(\sqrt{x}) \square 0.5 \delta(x-2) \quad[16$, Eq. (63)] instead to eliminate the need for integration involving coherent BPSK modulation. Whereas in [15], the same author suggested decomposing the integrand of $E\left[Q^{p}(x)\right]$ into a product of a generalized function $g(x)$ (i.e., "nascent" delta function) and an auxiliary function after replacing the $Q$-function with its approximation [15, Eq. (4)], and then simplifying the resulting integral by invoking an asymptotic Dirac delta approximation for $g(x)$ as (see Appendix B)

$$
g(x)=x^{c-1} \exp (-a x) \doteq \frac{\Gamma(c)}{a^{c}} \delta(x-c / a)
$$

where notation "ㄹ" denotes the Dirac delta approximation.

Nonetheless, the ASER analysis of digital modulations via Dirac delta approximation technique has thus far been restricted to coherent BPSK and differentially-encoded coherent BPSK and 
QPSK modulation schemes. Furthermore, recognizing that the shifting point can directly impact the overall accuracy of the resulting ASER approximation, we anticipate that the use of a tighter series approximation for $Q(x)$ (in lieu of [15, Eq. (4)]) may lead to an improved ASER approximation. Hence, one of the objectives of this paper is to extend [15] and [16] by deriving simple closed-form expressions for the ASER of a wide range of digital modulation schemes in conjunction with improved $Q$-function approximations given in [9, Eq. (25)] and [14]. In particular, our results in Section 2 generalize [15]-[16] to higher order signal constellations (i.e., may be utilized to predict the ASER of $M$-QAM, $M$-PSK as well as differentially encoded $M$ PSK) in addition to yielding slightly better approximations even for the specific cases considered in [15]. In Section 3, we also derive analytically simple and tight closed-form ASER approximations using the MGF method. While exponential-type approximations for $Q(x)$ presented in [4], [8] and [12]-[14] are already in a desirable exponential form, to the best of our knowledge, their utility in ASER analysis of DE-BPSK and DE-QPSK over generalized fading channels have not been reported previously. In the Appendix, we also develop a rapidly converging series expression for a generic integral via Gauss-Chebyshev quadrature (GCQ) numerical integration technique and subsequently highlight some of its application including the development of an efficient and asymptotically exact ASER formula for differentially-encoded $M$-PSK over fading channels via the MGF method. Selected computational results and comparisons between various ASER approximations for different $M$-ary modulation schemes and fading environments are provided in Section 4.

\section{Dirac Delta APPROXiMation}

Similar to [15] and [22], we consider a normalized probability density function (PDF) of the fading channel SNR in the form

$$
p_{\gamma}(\gamma)=K \exp (-h \gamma) \gamma^{c-1} f(\gamma), \gamma \geq 0
$$

where $\gamma$ denotes the squared magnitude of the channel fading amplitude, $K$ is a constant, and $f(\gamma)$ is an auxiliary function that depends on fading characteristics. The coefficients $K, h, c$ and $f(\gamma)$ for several different wireless channel models are also summarized in Table 2. From Table 1 , we can also write down a generic expression for the symbol error probability of a wide range of digital modulation schemes in AWGN as

$$
P_{s}(\gamma)=\sum_{z=1}^{Z} \alpha_{z} Q^{p_{z}}\left(\sqrt{\beta_{z} \Omega \gamma}\right)
$$

where $\alpha_{z}$ and $\beta_{z}$ are constants that depend on a specified digital modulation, and $\Omega=E_{s} / N_{0}$ corresponds to the received symbol SNR. To compute the ASER, we need to find the statistical expectation of (4) with respect to the fading random variable $\gamma$, viz.,

$$
\begin{aligned}
\overline{P_{s}} & =\sum_{z=1}^{Z} \alpha_{z} K \int_{0}^{\infty} Q^{p_{z}}\left(\sqrt{\beta_{z} \Omega \gamma}\right) \exp (-h \gamma) \gamma^{c-1} f(\gamma) d \gamma \\
& =\sum_{z=1}^{Z} \frac{\alpha_{z} K}{\left(\beta_{z} \Omega\right)^{c}} \int_{0}^{\infty} Q^{p_{z}}(\sqrt{x}) \exp \left(\frac{-h x}{\beta_{z} \Omega}\right) x^{c-1} f\left(\frac{x}{\beta_{z} \Omega}\right) d x
\end{aligned}
$$

In the remaining part of this section, we will consider various approximations for $Q(x)$ in (5), and simplify the integration task by exploiting the asymptotic Dirac delta approximation (2) with the shifting property of $\delta($.$) .$

\subsection{Jang's $Q$-function Approximation}

Expanding the integer powers of [15, Eq. (4)] using the binomial theorem, we obtain 


$$
\begin{aligned}
Q^{p_{z}}(x) & \cong \frac{\exp \left(-x^{2} p_{z} / 2\right)}{(2 \pi)^{p_{z} / 2}} \sum_{k=0}^{p_{z}}\left(\begin{array}{c}
p_{z} \\
k
\end{array}\right)\left(-\frac{\exp (-x-1 / 2)}{x+1}\right)^{k}\left(\frac{1}{x}\right)^{p_{z}-k} \\
& =\frac{\exp \left(-x^{2} p_{z} / 2\right)}{(2 \pi)^{p_{z} / 2}}\left[\frac{1}{x}-\frac{\exp (-x-0.5)}{x+1}\right]^{p_{z}}
\end{aligned}
$$

Substituting (6) into (5), and then simplifying the resulting expression using (2), we immediately arrive at

$$
\overline{P_{s}} \doteq \sum_{z=1}^{z} \frac{K \alpha_{z} \Gamma(c)}{(2 \pi)^{p_{z} / 2}\left(a_{z} \beta_{z} \Omega\right)^{c}} f\left(\frac{c}{a_{z} \beta_{z} \Omega}\right)\left[\sqrt{\frac{a_{z}}{c}}-\left(\sqrt{\frac{c}{a_{z}}}+1\right)^{-1} \exp \left(-\sqrt{\frac{c}{a_{z}}}-\frac{1}{2}\right)\right]^{p_{z}}
$$

where $a_{z}=p_{z} / 2+h /\left(\beta_{z} \Omega\right)$ and $\Gamma(c)=\int_{0}^{\infty} x^{c-1} e^{-x} d x$ denotes the Gamma function. For the readers convenience, we have also summarized the fading/modulation parameter selections in Table 2 (i.e., obtained by setting $b=1$ ). It is important to highlight that (7) generalizes [15, eq. (11)] to a broader class of digital modulation schemes (see Table 1).

\subsection{Boyd's $Q$-function Approximation}

In this subsection, we derive two new Dirac delta approximations for (5) based on Boyd's upper and lower bounds for $Q(x)$ [9, Eq. (25)]. Our work is motivated by the fact that these bounds are much tighter than [15, Eq. (4)] (especially near zero), and also due to their simple form that leads to upper and lower bounds for the integer powers of $Q(x)$ in an identical form, viz.,

$$
F^{p}(x, \pi-1) \leq Q^{p}(x) \leq F^{p}(x, 2 /(\pi-2))
$$

where the auxiliary function $F^{p}(x, \psi)$ is defined as

$$
F^{p}(x, \psi)=\left(\frac{(\psi+1) / \sqrt{2 \pi}}{\psi x+\sqrt{x^{2}+2(\psi+1)^{2} / \pi}}\right)^{p} \exp \left(-p x^{2} / 2\right)
$$

The lower bound is slightly tighter than the upper bound when the argument $x>1.23$ for $p=1$. Nevertheless, Dirac delta approximations for both the lower and the upper bounds can be obtained by an appropriate substitution for the coefficient $\psi$ in (9). Substituting (9) into (5), and then invoking the Dirac delta approximation (2), we obtain

$$
\overline{P_{s}} \doteq \sum_{z=1}^{z} \frac{K \alpha_{z} \Gamma(c)}{\left(a_{z} \beta_{z} \Omega\right)^{c}} f\left(\frac{c}{a_{z} \beta_{z} \Omega}\right)\left(\frac{(\psi+1) / \sqrt{2 \pi}}{\psi \sqrt{\frac{c}{a_{z}}}+\sqrt{\frac{c}{a_{z}}+\frac{2}{\pi}(\psi+1)^{2}}}\right)^{p_{z}}
$$

The coefficients required for evaluating (10) are provided in Table 2 (by setting $b=1$ ).

\subsection{Olabiyi's $Q$-function Approximation}

More recently, [13]-[14] have developed accurate and invertible exponential-type approximations (up to the third order) for $Q(x)$ by approximating the erfc(.) function as a weighted sum of powers of an exponential function. This form is particularly suitable for finding the statistical expectation of the CEP (including integer powers of $Q($.$) ) over the PDF of$ fading SNR. Hence in this subsection, we will investigate the efficacy of this new $Q(x)$ approximation for deriving simple and accurate closed-form ASER formulas for DE-BPSK and 
International Journal of Computer Networks \& Communications (IJCNC) Vol.5, No.1, January 2013

Table 2. Coefficients for various stochastic channel models.

\begin{tabular}{|c|c|c|c|c|c|}
\hline $\begin{array}{c}\text { Channel } \\
\text { Model }\end{array}$ & $K$ & $c$ & $h$ & $a$ & $f(c /(a \beta \Omega))$ \\
\hline Nakagami- $m$ & $m^{m} / \Gamma(m)$ & $m$ & $m$ & $\frac{b p}{2}+\frac{m}{\beta \Omega}$ & 1 \\
\hline Nakagami- $n$ & $\left(1+n^{2}\right) \exp \left(-n^{2}\right)$ & 1 & 1 & $\frac{b p}{2}+\frac{1}{\beta \Omega}$ & $\exp \left(-\frac{n^{2}}{a \beta \Omega}\right) I_{0}\left(2 n \sqrt{\frac{1+n^{2}}{a \beta \Omega}}\right)$ \\
\hline Nakagami- $q$ & $\left(1+q^{2}\right) /(2 q)$ & 1 & $\frac{\left(1+q^{2}\right)^{2}}{4 q^{2}}$ & $\frac{b p}{2}+\frac{\left(1+q^{2}\right)^{2}}{4 q^{2} \beta \Omega}$ & $I_{0}\left(\frac{1-q^{4}}{4 q^{2} a \beta \Omega}\right)$ \\
\hline
\end{tabular}

DE-QPSK via the Dirac delta approximation approach. Since the $Q(x)$ approximation in [15, Eq. (4)] contains two exponential terms, we will only consider the second order exponentialtype approximation for $Q(x)$ in our comparisons, viz., [14, Eq. (2) and Table 2]

$$
Q(\sqrt{x}) \cong \frac{w_{1}}{2} \exp \left(\frac{-b x}{2}\right)+\frac{w_{2}}{2} \exp (-b x)
$$

where $w_{1}=0.3017, w_{2}=0.4389$, and $b=1.0510$. It is also worth mentioning that the invertible property of (11) is not very critical in our current application, and thus other exponential-type approximations such as [8, Eq. (13c)] can be used, if desired. Using the binomial theorem expansion, it is quite straight-forward to show that

$$
Q^{p_{z}}(\sqrt{x}) \cong\left(\frac{w_{1}}{2}\right)^{p_{z}} \sum_{k=0}^{p_{z}}\left(\begin{array}{c}
p_{z} \\
k
\end{array}\right)\left(\frac{w_{2}}{w_{1}}\right)^{k} \exp \left(-\frac{x b\left(p_{z}+k\right)}{2}\right)
$$

Next substituting (12) into (5), and then simplifying the resulting expression using (2) and the shifting property of Dirac delta function, we arrive at

$$
\overline{P_{s}} \doteq \sum_{z=1}^{z} K \alpha_{z}\left(\frac{w_{1}}{2}\right)^{p_{z}} \sum_{k=0}^{p_{z}}\left(\begin{array}{c}
p_{z} \\
k
\end{array}\right)\left(\frac{w_{2}}{w_{1}}\right)^{k} \frac{\Gamma(c)}{\left(\overline{a_{z}} \beta_{z} \Omega\right)^{c}} f\left(\frac{c}{\overline{a_{z}} \beta_{z} \Omega}\right)
$$

where $\tilde{a}_{z}=b\left(p_{z}+k\right) / 2+h /\left(\beta_{z} \Omega\right)=a_{z}+b k / 2$. But a slightly more compact ASER approximation formula can be derived if we first decompose the exponential term in (12) into a product of two exponential terms (i.e., $\exp \left(-x b p_{z} / 2\right) \exp (-x b k / 2)$ that allows one to simplify the binomial sum) before invoking the Dirac delta approximation (2), viz.,

$$
\overline{P_{s}} \doteq \sum_{z=1}^{z} \frac{K \alpha_{z} \Gamma(c)}{\left(a_{z} \beta_{z} \Omega\right)^{c} 2^{p_{z}}} f\left(\frac{c}{a_{z} \beta_{z} \Omega}\right)\left[w_{1}+w_{2} \exp \left(-\frac{b c}{2 a_{z}}\right)\right]^{p_{z}}
$$

where the coefficients $K, a_{z}$ and $c$ for different fading environments are summarized in Table 2 (with $b=1.0510$ ).

\section{Moment Generating Function Method}

In this section, we present yet another method for deriving simple and tight closed-form approximations for the ASER of DE-BPSK and DE-QPSK over fading channels. Specifically, we take advantage of a tight exponential-type approximation for $Q^{p}(x)$ (see Eq. (12)) in (5) to express the ASER as a weighted sum of the MGF of SNR, viz., 
International Journal of Computer Networks \& Communications (IJCNC) Vol.5, No.1, January 2013

$$
\overline{P_{s}} \cong \sum_{z=1}^{Z} \alpha_{z} K\left(\frac{w_{1}}{2}\right)^{p_{z}} \sum_{k=0}^{p_{z}}\left(\begin{array}{c}
p_{z} \\
k
\end{array}\right)\left(\frac{w_{2}}{w_{1}}\right)^{k} \phi_{\gamma}\left(\frac{\Omega \beta_{z} b\left(p_{z}+k\right)}{2}\right)
$$

by recognizing that the resulting integral,

$$
\int_{0}^{\infty} e^{-s \gamma} p_{\gamma}(\gamma) d \gamma=\phi_{\gamma}(s)
$$

is simply the Laplace transform of the PDF of SNR. This result (15) is rather interesting especially considering that most prior work on ASER analysis of DE-QPSK/DE-BPSK with/without maximal-ratio diversity receiver (e.g., [5], [6] and [11]) are quite restrictive and limited to the Nakagami- $m$ channel with independent and identically distributed (i.i.d) fading statistics. In contrast, (15) can be readily applied to characterize the ASER of DE-QPSK/DEBPSK over a myriad of fading environments (including non-identically distributed fading link statistics) and diversity transceivers. Furthermore, in our case the ASER expressions are mostly expressed in terms of elementary functions. For instance, the MGF of SNR in a Nakagami- $m$ channel is given by $\phi_{\gamma}(s)=m^{m} /(m+s)^{m}$. Furthermore, the MGFs of SNR for a number of stochastic channel models are summarized in Table 3.

Although we recognize that the accuracy of a series approximation for $Q(x)$ can be improved by considering more number of terms in that series (e.g., [8, Eq. (13d)]), but its efficiency will be determined by the tightness combined with the number of terms in that series. Hence, we have also developed highly accurate and computationally efficient series approximations for integer powers of the $Q$-function and the CEP of differentially-encoded $M$-PSK in the Appendix A, with the aid of GCQ approximation and multinomial theorem. These asymptotically exact closed-form approximations are also in a desirable exponential form and thus will facilitate ASER analysis over generalized fading channels via the MGF method.

\section{NUMERICAL RESUltS}

In this section, selected numerical results are provided to investigate the efficacies of our new approximations (7), (10), (13), (14) and (15) for ASER analyses of several coherent modulations in a myriad of fading environments.

Figure 1 shows a comparison of various ASER approximations for 4-PSK in different Nakagami- $m$ fading channels. The exact performance curve is generated using [1, Eq. (5.78)]. It is apparent that (10), (13) and (14) performs considerably better than (7) when the channel experience more severe fading (i.e., smaller fading severity index $m$ ) especially at lower values of the mean channel SNR $\Omega$. The choice of $\psi$ in (8) (that corresponds to the upper and lower bounds) appears to cause only a negligible effect on the overall tightness of the final ASER approximations using (10). Furthermore, the curve corresponding to (15) (MGF method) is also quite close the exact ASER curve for a wide range of $m$ and $\Omega$.

In Figure 2, we investigate the accuracies of various Dirac delta approximations when applied to higher order constellations, since the prior work on $M$-PSK is restricted to only $M=2$. It is evident that the curves generated using (7) (i.e., direct generalization of [15]) virtually breaksdown at small values of $\Omega$ as constellation size $M$ increases. This can be attributed to the increasing relative error of [5, Eq. (4)] with decreasing value of its argument. As anticipated, (10) yields better approximation than (7) in this case. The results in this Figure 2 are also interesting because we have now demonstrated that it is possible to derive relatively simple and reasonably accurate closed-form approximations for $M$-ary modulations via the Dirac delta approximation technique. 
International Journal of Computer Networks \& Communications (IJCNC) Vol.5, No.1, January 2013

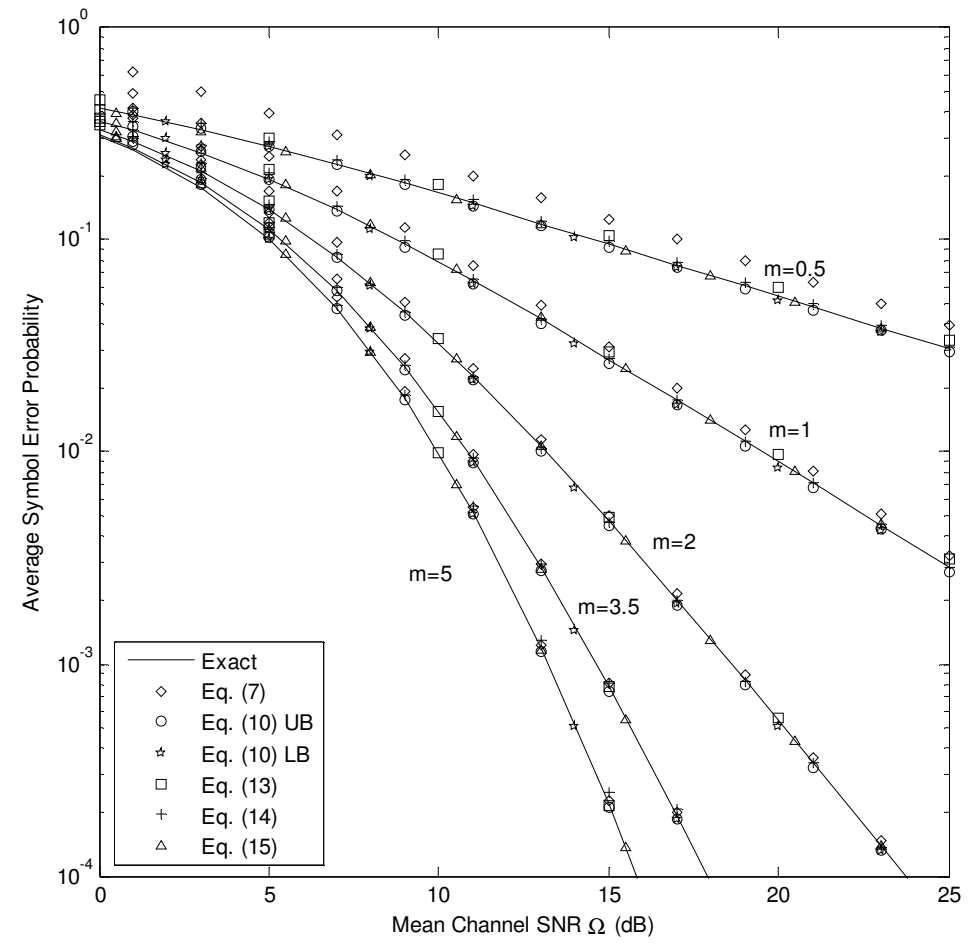

Figure 1. ASER of 4-PSK in Nakagami- $m$ fading $(m=0.5,1,2,3.5,5)$.

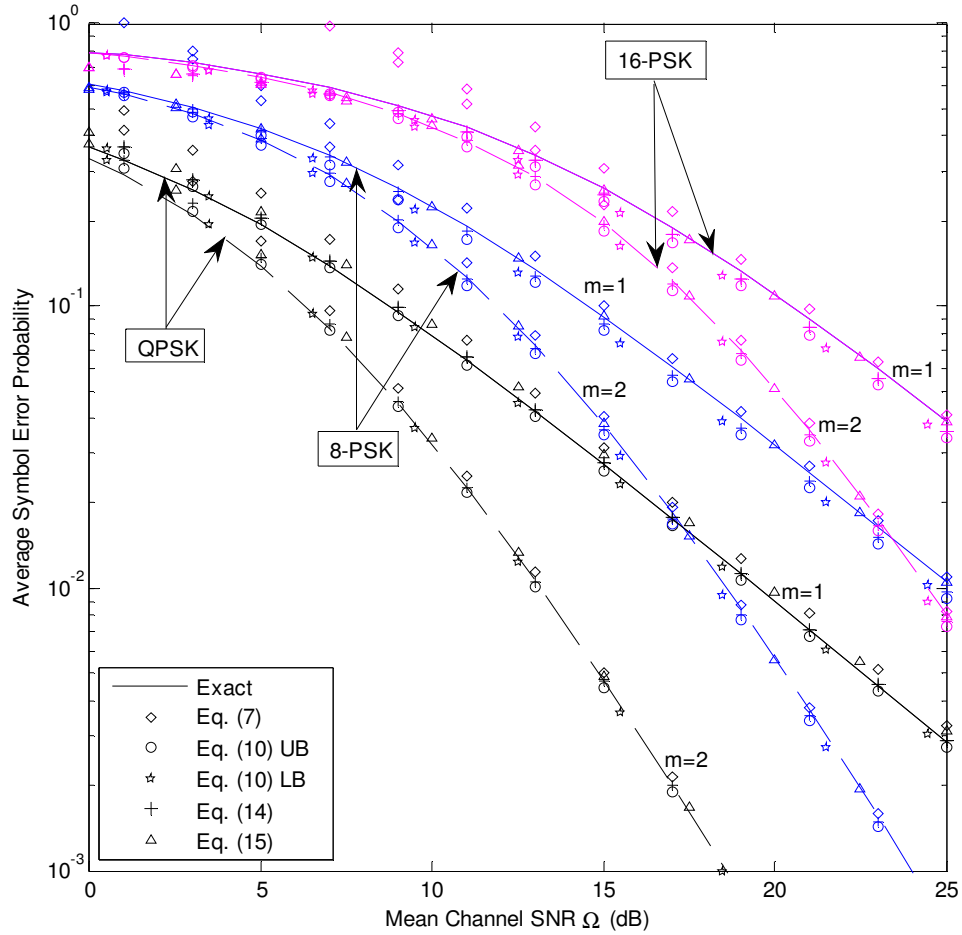

Figure 2. ASER of $M$-PSK $(M=4,8,16)$ in Nakagami- $m$ fading $(m=1,2)$. 
Table 3. MGF of SNR for several common stochastic channel models.

\begin{tabular}{|c|c|}
\hline Channel Model & $\begin{array}{c}\text { MGF of SNR } \\
\phi_{\gamma}(s)=\int_{0}^{\infty} f(\gamma) e^{-s \gamma} d \gamma\end{array}$ \\
\hline Rayleigh & $\left(1+s \Omega_{s}\right)^{-1}$ [1, Eq. (2.8)] \\
\hline $\begin{array}{l}\text { Nakagami- } n \\
\text { (Rice factor } K=n^{2} \text { ) }\end{array}$ & $\frac{1+K}{1+K+s \Omega_{s}} \exp \left(\frac{-K s \Omega_{s}}{1+K+s \Omega_{s}}\right) \quad[1$, Eq. (2.17)] \\
\hline Nakagami- $m$ & $\left(1+\frac{s \Omega_{s}}{m}\right)^{-m} \quad[1$, Eq. (2.22)] \\
\hline $\begin{array}{l}\text { Log-Normal } \\
\text { Shadowing }\end{array}$ & $\frac{1}{\sqrt{\pi}} \sum_{n=1}^{N_{p}} H_{x_{n}} \exp \left(-10^{\left(\sqrt{2} \sigma x_{n}+\mu\right) / 10} s\right) \quad[1$, Eq. $(2.54)]$ \\
\hline $\begin{array}{l}\text { Composite } \\
\text { Gamma/Log-Normal }\end{array}$ & $\frac{1}{\sqrt{\pi}} \sum_{n=1}^{N_{p}} H_{x_{n}}\left(1+10^{\left(\sqrt{2} \sigma x_{n}+\mu\right) / 10} s / m\right)^{-m} \quad[1$, Eq. (2.58)] \\
\hline$K$-Distribution & $\left(\frac{-v}{s \Omega_{s}}\right) \exp \left(\frac{-v}{s \Omega_{s}}\right) \Gamma\left(1-v, \frac{-v}{s \Omega_{s}}\right)[1$, Eq. (2.63)] \\
\hline$G$-Distribution & $\begin{array}{l}1+m \sum_{k=0}^{m-1}\left(\begin{array}{c}m-1 \\
k\end{array}\right) \frac{(-1)^{k+1}}{(k+1) !} \sum_{p=0}^{k}\left(\begin{array}{l}k \\
p\end{array}\right)(2 \sqrt{\alpha s / \beta})^{k+1-p} \\
\quad \times \Gamma(k+p+1) H_{-(k+p+1)}(0.5 b \sqrt{\beta / s}+\sqrt{\alpha s / \beta}) \quad[23 \text {, Eq. (17)] } \\
\text { where } \eta_{G}=\frac{\exp \left(\mu_{G}\right)}{2 \sinh \left(0.5 \sigma^{2}\right)}, \theta=\exp \left(\mu_{G}+0.5 \sigma^{2}\right), \beta=2 m \theta \text { and } \alpha=\eta_{G} \Omega_{s} .\end{array}$ \\
\hline Weibull & $\begin{array}{l}c\left(\frac{\Gamma(1+2 / c)}{\Omega_{s}}\right)^{c / 2}(2 \pi)^{(1-c) / 2} \frac{1}{\sqrt{c}}(s / c)^{-c} \\
\times G_{1, c}^{c, 1}\left(\left.\left(\frac{\Gamma(1+2 / c)}{\Omega_{s}}\right)^{-c / 2}(s / c)^{c}\right|_{1,1+1 / c, \ldots, 1+(c-1) / c}\right)\end{array}$ \\
\hline$\eta-\mu$ Distribution & $\left(\frac{4 \mu_{\eta}^{2} h}{\left(2(h-H) \mu_{\eta}+s \Omega_{s}\right)\left(2(h+H) \mu_{\eta}+s \Omega_{s}\right)}\right)^{\mu_{\eta}} \quad$ [24, Eq. (6)] \\
\hline$\kappa-\mu$ Distribution & $\left(\frac{\mu_{\kappa}(1+\kappa)}{\mu_{\kappa}(1+\kappa)+s \Omega_{s}}\right)^{\mu} \exp \left(\frac{\mu_{\kappa}^{2} \kappa(1+\kappa)}{\mu_{\kappa}(1+\kappa)+s \Omega_{s}}-\mu_{\kappa} \kappa\right) \quad[24$, Eq. (7)] \\
\hline
\end{tabular}

Note: Constants $K, m$ and $c$ correspond to the fading parameters of Rice, Nakagami and Weibull channels, $\mu(\mathrm{dB})$ and $\sigma(\mathrm{dB})$ are the mean and the standard deviation of $10 \log _{10} \Omega_{s}$ respectively, while $x_{n}$ and $H_{x_{n}}$ are the zeros and weight factors of the $N_{P}$-th order Hermite polynomial. Also the notation $G_{1, c}^{c, 1}($.$) denotes the Meijer's G-function, while \eta$ and $\kappa$ characterize the $\eta-\mu$ and $\kappa-\mu$ wireless fading channel models. 
Figure 3 depicts the ASER performance for DE-QPSK in different Nakagami- $m$ channels. It is interesting note that both (13) and (15) tend to yield very good ASER approximations over a wide range of $\Omega$ and $m$ values, compared to all other approximations. It is also evident that (7) becomes very accurate as $m$ increases. To investigate this trend further, in Table 4 we summarize the ASER values for various ASER approximations for DE-BPSK in Nakagami- $m$ fading. The trends observed from Figure 3 are also apparent from Table 4.

To apply Dirac delta approximation technique to Nakagami- $n$ and Nakagami- $q$ channels, it has been suggested in [15] to choose an auxiliary function as flat as possible so that the final ASER approximation will be robust to the sampling point error. The corresponding auxiliary functions are summarized in Table 2. At this point, we would like to emphasize that similar "ad-hoc" manipulations are not required for the MGF method discussed in Section 3. Furthermore, (15) can be applied directly (i.e., without any further manipulations) to study the multichannel reception case (e.g., maximal-ratio diversity, etc.). The same cannot be said for the Dirac delta approximation technique.

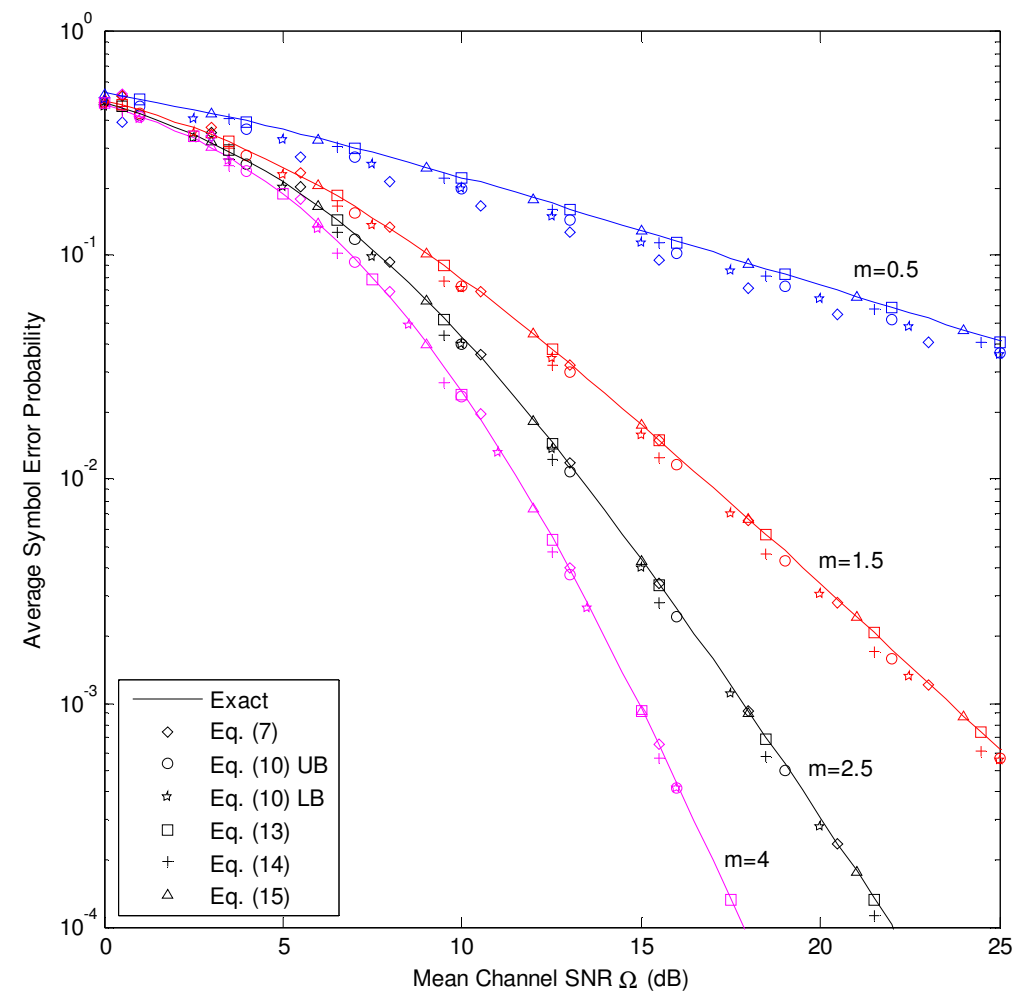

Figure 3. ASER of DE-QPSK in Nakagami- $m$ fading $(m=0.5,1.5,2.5,4)$.

The impact of fade distribution (i.e., fading parameter $n$ in the Nakagami- $n$ channel) and the constellation size of $M$-QAM on various ASER approximations developed in this paper are illustrated in Figure 4 and Figure 5, respectively. It is apparent that the accuracies of (7), (10) and (14) deteriorate with decreasing values of $n$ and $\Omega$. However, ASER approximations (10) and (14) are slightly better than (7). These trends are somewhat consistent with our observations for $M$-PSK in Nakagami- $m$ fading (see Figure 1 and Figure 2). Interestingly, the curves generated via closed-form approximation (15) are virtually indistinguishable compared to their exact performance curves obtained using an integral formula in [1]. 
International Journal of Computer Networks \& Communications (IJCNC) Vol.5, No.1, January 2013

Table 4. ASER of DE-BPSK in Nakagami- $m$ fading $(m=0.5,3)$.

\begin{tabular}{|l|l|l|l|l|l|}
\hline \multicolumn{7}{|c|}{$\Omega$} & $0 \mathrm{~dB}$ & $5 \mathrm{~dB}$ & $10 \mathrm{~dB}$ & $20 \mathrm{~dB}$ & $25 \mathrm{~dB}$ \\
\hline \multicolumn{7}{|c|}{ Fading severity index $m=0.5$} \\
\hline Exact & $2.677 \mathrm{e}-1$ & $1.683 \mathrm{e}-1$ & $9.863 \mathrm{e}-2$ & $3.176 \mathrm{e}-2$ & $1.789 \mathrm{e}-2$ \\
\hline Eq. (7) & $2.236 \mathrm{e}-1$ & $1.381 \mathrm{e}-1$ & $8.008 \mathrm{e}-2$ & $2.565 \mathrm{e}-2$ & $1.444 \mathrm{e}-2$ \\
\hline Eq. (10) UB & $2.183 \mathrm{e}-1$ & $1.425 \mathrm{e}-1$ & $8.500 \mathrm{e}-2$ & $2.761 \mathrm{e}-2$ & $1.556 \mathrm{e}-2$ \\
\hline Eq. (10) LB & $2.159 \mathrm{e}-1$ & $1.408 \mathrm{e}-1$ & $8.398 \mathrm{e}-2$ & $2.728 \mathrm{e}-2$ & $1.537 \mathrm{e}-2$ \\
\hline Eq. (13) & $2.634 \mathrm{e}-1$ & $1.659 \mathrm{e}-1$ & $9.727 \mathrm{e}-2$ & $3.133 \mathrm{e}-2$ & $1.765 \mathrm{e}-2$ \\
\hline Eq. (14) & $2.286 \mathrm{e}-1$ & $1.497 \mathrm{e}-1$ & $8.909 \mathrm{e}-2$ & $2.888 \mathrm{e}-2$ & $1.627 \mathrm{e}-2$ \\
\hline Eq. (15) & $2.634 \mathrm{e}-1$ & $1.659 \mathrm{e}-1$ & $9.727 \mathrm{e}-2$ & $3.133 \mathrm{e}-2$ & $1.765 \mathrm{e}-2$ \\
\hline \multicolumn{7}{|c|}{ Fading severity index $m=3$} \\
\hline Exact & $1.770 \mathrm{e}-1$ & $4.211 \mathrm{e}-2$ & $4.027 \mathrm{e}-3$ & $7.537 \mathrm{e}-6$ & $2.515 \mathrm{e}-7$ \\
\hline Eq. (7) & $2.021 \mathrm{e}-1$ & $4.460 \mathrm{e}-2$ & $4.112 \mathrm{e}-3$ & $7.553 \mathrm{e}-6$ & $2.517 \mathrm{e}-7$ \\
\hline Eq. (10) UB & $3.470 \mathrm{e}-2$ & $1.340 \mathrm{e}-2$ & $2.160 \mathrm{e}-3$ & $6.498 \mathrm{e}-6$ & $2.281 \mathrm{e}-7$ \\
\hline Eq. (10) LB & $3.423 \mathrm{e}-2$ & $1.321 \mathrm{e}-2$ & $2.133 \mathrm{e}-3$ & $6.428 \mathrm{e}-6$ & $2.257 \mathrm{e}-7$ \\
\hline Eq. (13) & $1.802 \mathrm{e}-1$ & $4.181 \mathrm{e}-2$ & $3.955 \mathrm{e}-3$ & $7.399 \mathrm{e}-6$ & $2.470 \mathrm{e}-7$ \\
\hline Eq. (14) & $3.495 \mathrm{e}-2$ & $1.279 \mathrm{e}-2$ & $1.981 \mathrm{e}-3$ & $6.029 \mathrm{e}-6$ & $2.124 \mathrm{e}-7$ \\
\hline Eq. (15) & $1.802 \mathrm{e}-1$ & $4.181 \mathrm{e}-2$ & $3.955 \mathrm{e}-3$ & $7.399 \mathrm{e}-6$ & $2.470 \mathrm{e}-7$ \\
\hline
\end{tabular}

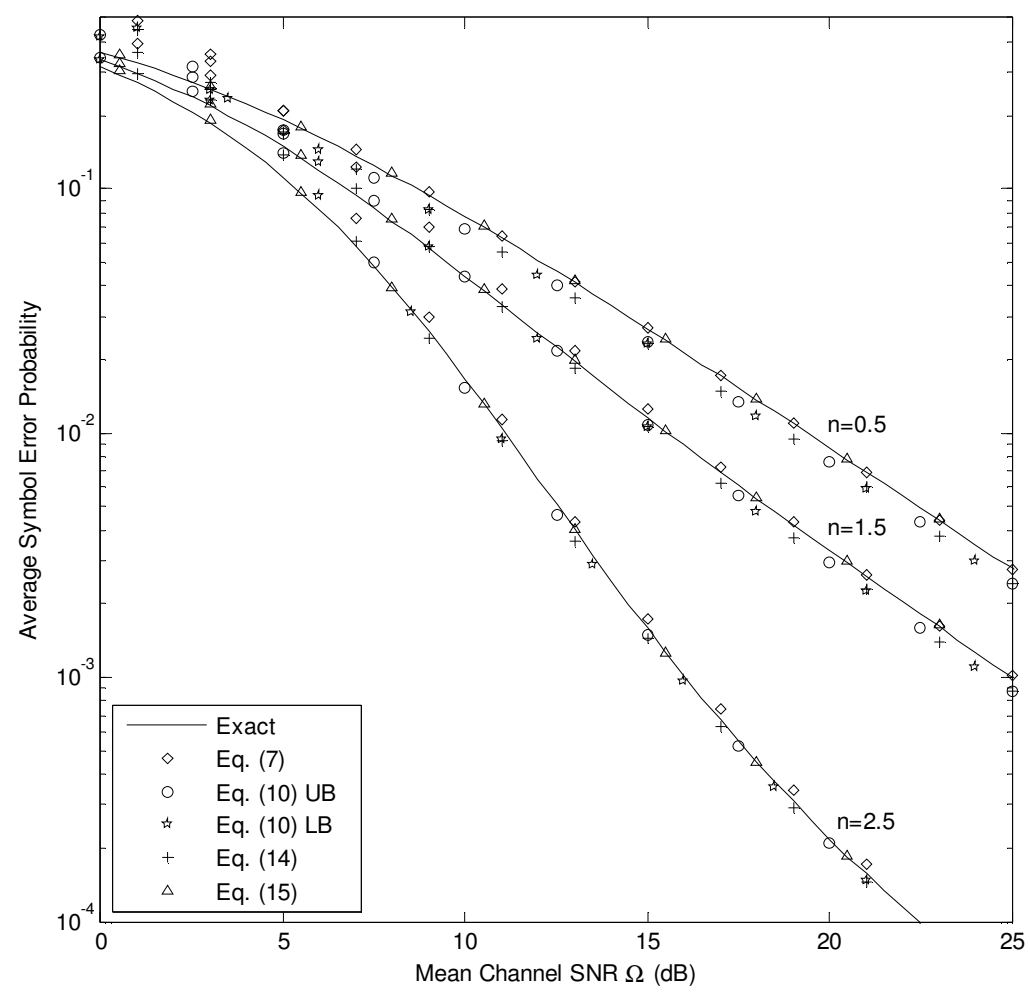

Figure 4. ASER of 4-QAM in Nakagami- $n$ fading $(n=0.5,1.5,2.5)$. 


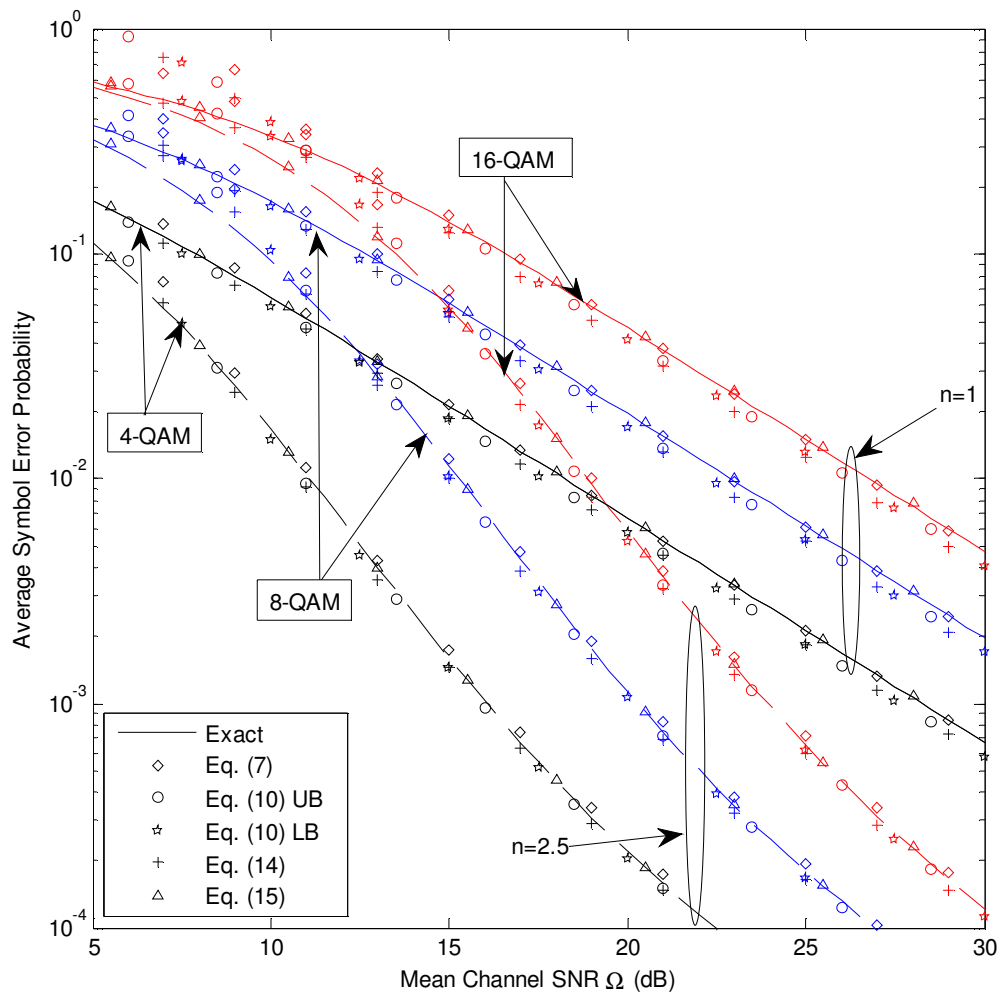

Figure 5. ASER of $M$-QAM in Nakagami- $n$ fading $(n=1,2.5)$.

\section{CONCLUSIONS}

In this article, we have studied two novel methods for efficient evaluation of ASER for a broad class of coherently detected digital modulation schemes in Nakagami- $m$, Nakagami- $n$ and Nakagami- $q$ channels. Our new closed-form Dirac delta approximations achieves better accuracy than those reported in [15] and [16], besides generalizing their results to higher order constellations. In addition, we have highlighted the advantages and limitations of Dirac delta approximation method for ASER analysis. We have also demonstrated that our closed-form ASER approximation based on the MGF method (15) is highly accurate and is more versatile than all other asymptotic Dirac delta approximations. In the Appendix, we have shown that asymptotically exact ASER and/or average block error rate in fading channels can be computed efficiently by exploiting a Gaussian quadrature method in conjunction with multinomial theorem, albeit at additional computational cost.

\section{APPENDiX A}

In this appendix, we will consider evaluating a generic integral of the form depicted in (A.1) via Gauss-Chebyshev quadrature (GCQ) numerical integration technique. This approach leads to a rapidly converging series approximation for the integral besides circumventing the need for a look-up table to store the weights and abscissas associated with other variants of Gaussian quadrature methods. Although a small number of integration points are sufficient to yield a good accuracy, one may readily increase the order $N$ to satisfy a prescribed relative error without any difficulty since the weights and abscissas for the GCQ approximation are in closedform. Interestingly, this result can be also utilized to derive exponential-type series approximations for higher integer powers of the Gaussian probability integral $Q(x)$ and other related functions. 
Let us consider a generic integral of the form

$$
g[\delta, \alpha, \beta]=\int_{0}^{\alpha \pi} \delta f(\beta, \theta) d \theta
$$

where the integrand is a function of the integration variable $\theta$ and constants $\delta$ and $\beta$. Applying the variable substitution $t=\cos (\theta / \alpha)$ (i.e., $\theta=\alpha \cos ^{-1}(t)$ and $d \theta=-d t \alpha / \sqrt{1-t^{2}}$ ) in (A.1), we obtain

$$
g[\delta, \alpha, \beta]=\alpha \delta \int_{-1}^{1} \frac{f\left(\beta, \alpha \cos ^{-1}(t)\right)}{\sqrt{1-t^{2}}} d t
$$

It is now straight-forward to evaluate (A.2) using the GCQ method with abscissas $\cos [(2 k-1) \pi /(2 N)]$ (which are zeros of the $N^{\text {th }}$ degree Chebyshev polynomial of the first kind) and weights $\pi / N$, viz.,

$$
g[\delta, \alpha, \beta] \approx \frac{\alpha \delta \pi}{N} \sum_{k=1}^{N} f\left(\beta, \frac{\alpha(2 k-1) \pi}{2 N}\right)
$$

Next, we will present several applications of (A.3) to simplify the evaluation of the integral in the form of (A.1).

Example 1:

In the first example, we will highlight the utility of (A.3) for deriving simple closed-from ASER approximations for $M$-ary phase shift keying ( $M$-PSK) and $M$-ary differential phase shift keying ( $M$-DPSK) modulation schemes over generalized stochastic fading environments. Using the MGF approach for performance analysis of $M$-PSK with diversity receivers, we can show that the exact ASER is given by [1, Eq. (9.15)]

$$
\bar{P}_{s}=\frac{1}{\pi} \int_{0}^{(M-1) \pi / M} \phi_{\gamma}\left(\frac{\Omega \sin ^{2}(\pi / M)}{\sin ^{2}(\theta)}\right) d \theta
$$

where $\phi_{\gamma}(s)$ denotes the MGF of SNR in a specified fading environment. Substituting $\alpha=(M-1) / M, \beta=\Omega \sin ^{2}(\pi / M), \delta=1 / \pi$ and $f(\beta, \theta)=\phi_{\gamma}\left(\beta \csc ^{2}(\theta)\right)$ in (A.1), we get

$$
\bar{P}_{s} \approx \frac{M-1}{M N} \sum_{k=1}^{N} \phi_{\gamma}\left(\frac{\Omega \sin ^{2}(\pi / M)}{\sin ^{2}((M-1)(2 k-1) \pi /(2 N M))}\right)
$$

Mimicking the above steps, we can also derive a closed-form approximation for the ASER of $M$-DPSK [1, Eq. (8.200)] as

$$
\bar{P}_{s} \approx \frac{M-1}{M N} \sum_{k=1}^{N} \phi_{\gamma}\left(\frac{\Omega \sin ^{2}(\pi / M)}{1+\cos (\pi / M) \cos ((M-1)(2 k-1) \pi /(2 N M))}\right)
$$

The above technique may be readily used to simplify the performance evaluation of any arbitrary two-dimensional signal constellations with/without diversity receivers, but the details are omitted here for brevity.

Example 2:

In our second example, we will employ (A.3) to derive simple exponential-type approximations for $Q(x)$ and its integer powers. One of the benefits of applying GCQ approximation to the conditional error probability (prior to taking statistical expectations over the fading density functions) when dealing with the powers of $g[\delta, \alpha, \beta]$ is that multinomial theorem can be 
exploited to minimize the number of resulting summation terms compared to simplifying the resulting multi-fold integral after performing the statistical averaging over the fading SNR. This result is of interest in the ASER analysis of differentially-encoded $M$-PSK schemes over fading channels. For instance, the CEP of coherently detected DE-BPSK and DE-QPSK are depicted in (A.7) and (A.8) respectively:

$$
\begin{gathered}
P_{s}=2 Q(\sqrt{2 \Omega \gamma})-2 Q^{2}(\sqrt{2 \Omega \gamma}) \\
P_{s}=4 Q(\sqrt{\Omega \gamma})-8 Q^{2}(\sqrt{\Omega \gamma})+8 Q^{3}(\sqrt{\Omega \gamma})-4 Q^{4}(\sqrt{\Omega \gamma})
\end{gathered}
$$

Substituting $\alpha=1 / 2, \beta=x^{2} / 2, f(\beta, \theta)=\exp \left(-\beta \csc ^{2}(\theta)\right)$ and $\delta=1 / \pi$ [2, Eq. (4.2)] in (A.3), we get a rapidly converging exponential-type series approximation for $Q(x)$, viz.,

$$
Q(x) \approx \frac{1}{2 N} \sum_{k=1}^{N} \exp \left[\frac{-x^{2}}{2 \sin ^{2}\left(\frac{\pi}{4 N}(2 k-1)\right)}\right]
$$

It is important to highlight that the above series converges to its exact value considerably faster than the corresponding Reinmann integration sum presented in [4, Eq. (8)]. Besides, it completely eliminates the need for finding the optimized coefficients in the Prony's approximation and their associated computational difficulties (e.g., selection of the range as well as the data points for "curve-fitting") especially for higher order approximations (thereby, providing a greater flexibility for attaining a prescribed accuracy).

Exponential-type approximations for higher integer powers $(p \geq 1)$ of Gaussian probability integral can be attained via multinomial expansion of (A.9), i.e.,

$$
Q^{p}(x) \approx \frac{1}{(2 N)^{p}} \sum_{k_{1}+\ldots+k_{N}=p}\left(\begin{array}{c}
p \\
k_{1}, \ldots, k_{N}
\end{array}\right) \exp \left[-x^{2} \sum_{t=1}^{N} \frac{k_{t}}{2 \sin ^{2}\left(\frac{\pi}{4 N}(2 t-1)\right)}\right]
$$

where $\left(\begin{array}{c}p \\ k_{1}, k_{2}, \ldots, k_{N}\end{array}\right)=\frac{p !}{k_{1} ! k_{2} ! \ldots k_{N} !}$ and $k_{i} \in\{0,1, \ldots, p\}$. The number of terms in the multinomial sum is given by $\frac{(p+N-1) !}{p !(N-1) !}$. Hence it is apparent that (A.10) requires significantly fewer number summation terms compared to repeatedly multiplying (A.9) to achieve the same level of accuracy. For instance, when $N=5$ and $p=3$, the number of summation terms in (A.10) and that of repeatedly multiplying (A.9) are given by $7 ! /(3 ! 4 !)=35$ and $5^{3}=125$, respectively. Nevertheless, a much simpler closed-form GCQ approximation for $Q^{2}(x), Q^{3}(x)$ and $Q^{4}(x)$ can be derived from their respective single exponential-type integral representation depicted in $[1$, Eq. (4.9)], [1, Eq. (4.31)] and [1, Eq. (4.32)]. The results for $Q^{2}(x)$ and $Q^{4}(x)$ are summarized below as illustrative examples:

$$
\begin{gathered}
Q^{2}(x) \approx \frac{1}{4 N} \sum_{k=1}^{N} \exp \left[\frac{-x^{2}}{2 \sin ^{2}\left(\frac{\pi}{8 N}(2 k-1)\right)}\right] \\
Q^{4}(x) \approx \frac{1}{6 \pi N} \sum_{k=1}^{N} \cos ^{-1}\left[\frac{3 \cos \left(\frac{\pi}{6 N}(2 k-1)\right)-1}{2 \cos ^{3}\left(\frac{\pi}{6 N}(2 k-1)\right)}-1\right] \exp \left[\frac{-x^{2}}{2 \sin ^{2}\left(\frac{\pi}{12 N}(2 k-1)\right)}\right]
\end{gathered}
$$

Hence a simple exponential-type approximation for $Q^{5}(x)$ can be obtained by multiplying the series approximations (A.12) and (A.9). It is evident that the resulting series approximation is simpler than (A.10). It is also important to recognize that a rapidly converging exponential-type series approximation for (A.8) can be obtained with the aid of [1, Eqs. (4.2), (4.9), (4.31)(4.32)] and (A.3), viz., 
International Journal of Computer Networks \& Communications (IJCNC) Vol.5, No.1, January 2013

$$
\begin{aligned}
P_{s} \approx & \frac{2}{N} \sum_{k=1}^{N} \exp \left[\frac{-\Omega \gamma}{2 \sin ^{2}\left(\frac{\pi}{4 N}(2 k-1)\right)}\right]-\frac{2}{N} \sum_{k=1}^{N} \exp \left[\frac{-\Omega \gamma}{2 \sin ^{2}\left(\frac{\pi}{8 N}(2 k-1)\right)}\right] \\
+ & \frac{2}{3 N \pi} \sum_{k=1}^{N} \cos ^{-1}\left[\frac{3 \cos \left(\frac{\pi}{6 N}(2 k-1)\right)-1}{2 \cos ^{3}\left(\frac{\pi}{6 N}(2 k-1)\right)}-1\right] \exp \left[\frac{-\Omega \gamma}{2 \sin ^{2}\left(\frac{\pi}{12 N}(2 k-1)\right)}\right] \\
+ & \frac{4 \sin ^{-1}(1 / \sqrt{3})}{N \pi^{2}} \sum_{k=1}^{N} \exp \left[\frac{-\Omega \gamma}{2 \sin ^{2}\left(\sin ^{-1}(1 / \sqrt{3})(2 k-1) /(2 N)\right)}\right] \\
& \times\left\{\pi-\cos ^{-1}\left[\frac{3 \cos \left(\sin ^{-1}(1 / \sqrt{3})(2 k-1) / N\right)-1}{2 \cos ^{3}\left(\sin ^{-1}(1 / \sqrt{3})(2 k-1) / N\right)}-1\right]\right\}
\end{aligned}
$$

The above result is quite interesting in that several researchers had in the last five years developed various non-exponential type approximations for $Q(x)$ and subsequently applied their approximations to derive closed-form approximations for the ASER of DE-QPSK modulation with maximal-ratio diversity receiver (e.g., [5], [6] and [11]). However, their results were restricted to Nakagami- $m$ channels with independent and identically distributed (i.i.d) fading statistics. In contrast, our closed-form approximation (A.13) can be readily applied to characterize the ASER of DE-QPSK modulation over a myriad of wireless fading channels (including non-identically distributed fading link statistics).

\section{Example 3:}

In our third example, we will demonstrate the efficacy of (A.3) and its multinomial expansion for facilitating the ASER analysis of coherently detected differentially-encoded $M$-PSK particularly when the constellation size $M$ is greater than 4 . In this case, simplifications of the conditional error probability similar to (A.7), (A.8) or (A.13) do not seem feasible. If we define $f(\beta, \theta)=\exp \left(-\beta / \sin ^{2}(\theta)\right)$ in (A.1), then the desired symbol error probability in an AWGN channel [1, Eq. (8.36)] can be expressed as

$$
\begin{aligned}
P_{s}=2 g[ & \left.\frac{1}{\pi}, \frac{M-1}{M}, \Omega \gamma \sin ^{2}\left(\frac{\pi}{M}\right)\right]-g^{2}\left[\frac{1}{\pi}, \frac{M-1}{M}, \Omega \gamma \sin ^{2}\left(\frac{\pi}{M}\right)\right] \\
- & \sum_{j=1}^{M-1}\left\{\frac{1}{4} g^{2}\left[\frac{1}{\pi}, 1-\frac{2 j-1}{M}, \Omega \gamma \sin ^{2}\left(\frac{(2 j-1) \pi}{M}\right)\right]+\frac{1}{4} g^{2}\left[\frac{1}{\pi}, 1-\frac{2 j+1}{M}, \Omega \gamma \sin ^{2}\left(\frac{(2 j+1) \pi}{M}\right)\right]\right. \\
& \left.-\left(\frac{1}{2} g\left[\frac{1}{\pi}, 1-\frac{2 j-1}{M}, \Omega \gamma \sin ^{2}\left(\frac{(2 j-1) \pi}{M}\right)\right] g\left[\frac{1}{\pi}, 1-\frac{2 j+1}{M}, \Omega \gamma \sin ^{2}\left(\frac{(2 j+1) \pi}{M}\right)\right]\right)\right\}
\end{aligned}
$$

As highlighted in [1, pp. 235] the fact that the second and third terms of (A.14) involve squaring of integrals still poses some difficulties in terms of their extension to the fading channel. Hence the application of (A.3) in conjunction with the multinomial theorem [20] is particularly attractive in this case since it can overcome the above-mentioned issue. In fact, it is rather straight-forward to obtain GCQ approximations for $g[1 / \pi, \alpha, \beta]$ and $g^{p}[1 / \pi, \alpha, \beta]$, viz.,

$$
\begin{gathered}
g\left[\frac{1}{\pi}, \alpha, \beta\right] \approx \frac{\alpha}{N} \sum_{k=1}^{N} \exp \left[\frac{-\beta}{\sin ^{2}\left(\frac{\pi}{2 N}(2 k-1) \alpha\right)}\right] \\
g^{p}\left[\frac{1}{\pi}, \alpha, \beta\right] \approx\left(\frac{\alpha}{N}\right)^{p} \sum_{k_{1}+\ldots+k_{N}=p}\left(\begin{array}{c}
p \\
k_{1}, \ldots, k_{N}
\end{array}\right) \exp \left[-\beta \sum_{t=1}^{N} \frac{k_{t}}{\sin ^{2}\left(\frac{\pi}{2 N}(2 t-1) \alpha\right)}\right]
\end{gathered}
$$

When $p=2$, there will be a total of $N(N+1) / 2$ terms in the above multinomial sum. It is also apparent that (A.15) and (A.16) are in a desirable exponential form and therefore, will facilitate 
International Journal of Computer Networks \& Communications (IJCNC) Vol.5, No.1, January 2013

statistical averaging over the density function of the fading SNR in a myriad of fading environments. Thus the ASER of DE-MPSK (for any positive integer $M$ which is a power of 2) can be expressed similarly as (A.14) yielding

$$
\begin{aligned}
\overline{P_{s}}= & 2 \bar{g}\left[\frac{1}{\pi}, \frac{M-1}{M}, \sin ^{2}\left(\frac{\pi}{M}\right)\right]-\overline{g^{2}}\left[\frac{1}{\pi}, \frac{M-1}{M}, \sin ^{2}\left(\frac{\pi}{M}\right)\right] \\
& -\sum_{j=1}^{M-1}\left\{\frac{1}{4} g^{2}\left[\frac{1}{\pi}, 1-\frac{2 j-1}{M}, \sin ^{2}\left(\frac{(2 j-1) \pi}{M}\right)\right]+\frac{1}{4} \bar{g}^{2}\left[\frac{1}{\pi}, 1-\frac{2 j+1}{M}, \sin ^{2}\left(\frac{(2 j+1) \pi}{M}\right)\right]\right. \\
& \left.-\frac{1}{2} \int_{0}^{\infty}\left(g\left[\frac{1}{\pi}, 1-\frac{2 j-1}{M}, \sin ^{2}\left(\frac{(2 j-1) \pi}{M}\right)\right] g\left[\frac{1}{\pi}, 1-\frac{2 j+1}{M}, \sin ^{2}\left(\frac{(2 j+1) \pi}{M}\right)\right]\right) f_{\gamma}(\gamma) d \gamma\right\}
\end{aligned}
$$

where

$$
\bar{g}\left[\frac{1}{\pi}, \alpha, \beta\right]=\int_{0}^{\infty} g\left[\frac{1}{\pi}, \alpha, \beta\right] f_{\gamma}(\gamma) d \gamma \approx \frac{\alpha}{N} \phi_{\gamma}\left[\beta \sum_{t=1}^{N} \frac{k_{t}}{\sin ^{2}\left(\frac{\pi}{2 N}(2 t-1) \alpha\right)}\right]
$$

$\phi_{\gamma}(s)$ denotes the MGF of SNR, $N$ corresponds to the number of GCQ terms, while the last term of (A.17) can be evaluated as

$$
\begin{aligned}
\overline{g_{1} g_{2}} & =\int_{0}^{\infty} g\left[\frac{1}{\pi}, \alpha_{1}, \beta_{1}\right] g\left[\frac{1}{\pi}, \alpha_{2}, \beta_{2}\right] f(\gamma) d \gamma \\
& \approx \frac{\alpha_{1} \alpha_{2}}{N^{2}} \sum_{k=1}^{N} \sum_{i=1}^{N} \phi_{\gamma}\left[\frac{-\beta_{1}}{\sin ^{2}\left(\frac{\pi}{2 N}(2 k-1) \alpha_{1}\right)}+\frac{-\beta_{2}}{\sin ^{2}\left(\frac{\pi}{2 N}(2 i-1) \alpha_{2}\right)}\right] .
\end{aligned}
$$

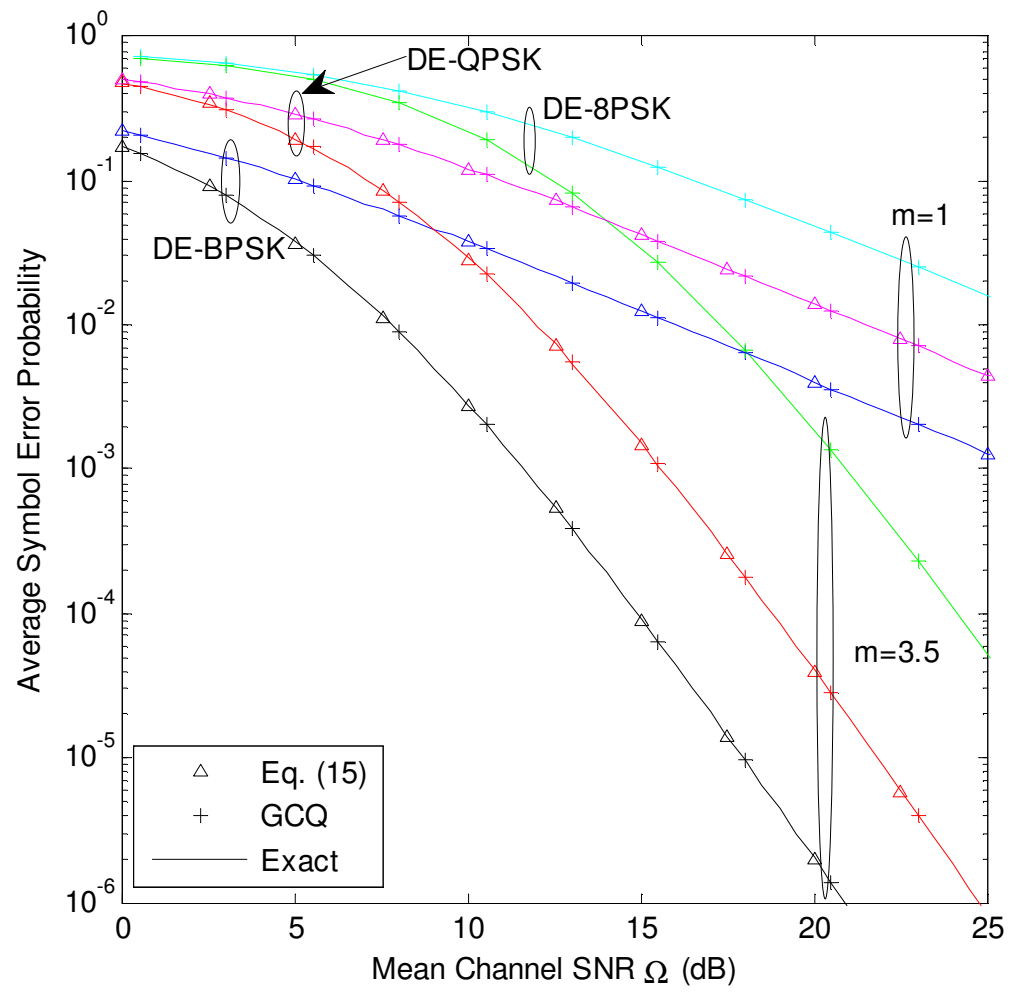

Figure 6. ASER of DE-MPSK $(M=2,4,8)$ in Nakagami- $m$ fading $(m=1,3.5)$. 
International Journal of Computer Networks \& Communications (IJCNC) Vol.5, No.1, January 2013

In Figure 6, we investigate the accuracies of the GCQ approximation (A.17) when applied to evaluate the ASER of DE-MPSK in different Nakagami- $m$ channels. It is interesting to note that the GCQ approximation can be applied to larger constellation sizes (i.e., $M>4$ ) while existing methods/results are restricted to only DE-BPSK and DE-QPSK.

\section{Appendix B}

In this appendix, we will show that if the integrand of $E_{\gamma}\left[Q^{p}(\sqrt{\beta \gamma})\right]=\int_{0}^{\infty} Q^{p}(\sqrt{\beta \gamma}) p_{\gamma}(\gamma) d \gamma$ can be decomposed into a product of a generalized function $g(\gamma)=\gamma^{c-1} \exp (-a \gamma)$ and an auxiliary function $\Delta(\gamma)$, then the Dirac delta approximation for $E_{\gamma}\left[Q^{p}(\sqrt{\beta \gamma})\right] \square \frac{\Gamma(c)}{a^{c}} \Delta(c / a)$. This is equivalent to invoking the Dirac delta approximation for $g(\gamma)$ as shown in (2).

Proof:

The Dirac delta function $\delta(x)$ can be defined as a limit of a class of "nascent" delta functions $\delta_{N}(x)$, and has the fundamental property that

$$
\operatorname{limit}_{N \rightarrow \infty} \int_{-\infty}^{\infty} \Delta(x) \delta_{N}(x) d x=c_{0} \int_{-\infty}^{\infty} \Delta(x) \delta\left(x-x_{0}\right) d x=c_{0} \Delta\left(x_{0}\right)
$$

where $c_{0}$ and $x_{0}$ are constants. In the following, we will consider a "nascent" delta function of the form $\delta_{N}(x)=N x^{c N-1} \exp \left(-a x^{N}\right)=g\left(x^{N}\right) N x^{N-1}$. Using variable substitution $\gamma=x^{N}$ in $\int_{0}^{\infty} g(\gamma) \Delta(\gamma) d \gamma$, we obtain

$$
\int_{0}^{\infty} g(\gamma) \Delta(\gamma) d \gamma=\int_{0}^{\infty} \delta_{N}(x) \Delta\left(x^{N}\right) d x
$$

It is evident from Figure 7 that for fixed values of $c$ and $a, \delta_{N}(x)$ quickly approaches the shape of an impulse function $\delta(x)$ as the value of $N$ gets larger.

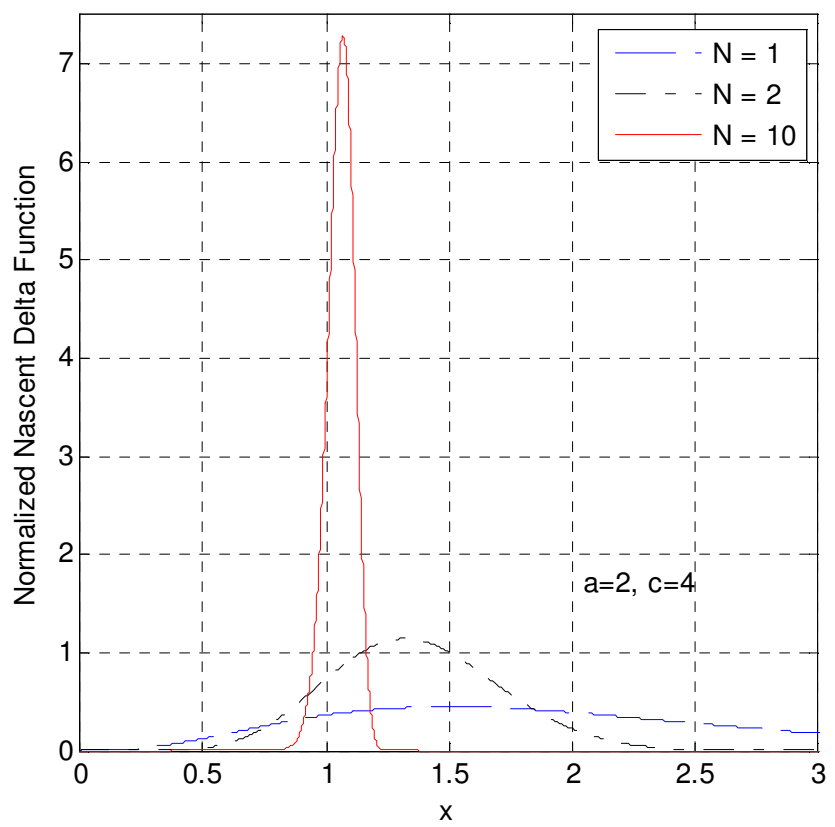

Figure 7. Normalized "nascent" delta function $\delta_{N}(x) a^{c} / \Gamma(c)$ as a function of $x$. 
In the following, we will show that

$$
\begin{aligned}
\operatorname{limit}_{N \rightarrow \infty} \delta_{N}(x) & =\operatorname{limit}_{N \rightarrow \infty} \frac{\Gamma(c)}{a^{c}} \delta\left(x-\left(\left(c-N^{-1}\right) / a\right)^{1 / N}\right) \\
& =\frac{\Gamma(c)}{a^{c}} \delta\left(x^{N}-c / a\right)
\end{aligned}
$$

To do so, we first recognize that

$$
c_{0}=\int_{0}^{\infty} \delta_{N}(x) d x=\int_{0}^{\infty} g(\gamma) d \gamma=\Gamma(c) / a^{c}
$$

Next, the shifting point for the Dirac delta approximation of $\delta_{N}(x)$ is obtained by solving $\frac{d}{d x} \delta_{N}(x)=0$ for $x$, viz.,

$$
\frac{d}{d x} \delta_{N}(x)=N \exp \left(-a x^{N}\right) x^{c N-2}\left[c N-1-a N x^{N}\right]=0
$$

This leads to $x_{0}=\left[\left(c-N^{-1}\right) / a\right]^{1 / N}$. As $N \rightarrow \infty$, we immediately arrive at (B.3). Also, substituting (B.3) into (B.2), we obtain $\int_{0}^{\infty} g(\gamma) \Delta(\gamma) d \gamma \square \frac{\Gamma(c)}{a^{c}} \Delta(c / a)$. This completes the proof.

Next, we will highlight the conditions for which the Dirac delta approximation technique will yield satisfactory results. From (B.1) and Figure 7, it is evident that the mass (i.e., most of the random values) of $X$ is concentrated around its mean $E(X)=x_{0}$. Thus, the tangent line that passes through coordinate $\left(x_{0}, \Delta\left(x_{0}\right)\right)$ with slope $\Delta^{\prime}\left(x_{0}\right)=\left.\frac{d}{d x} \Delta(x)\right|_{x=x_{0}} \quad$ is given by $Y=\Delta^{\prime}\left(x_{0}\right) X+\Delta\left(x_{0}\right)-\Delta^{\prime}\left(x_{0}\right) x_{0}$. An approximation to the same tangent line can be derived by using a Taylor series expansion of $\Delta(x)$ (to the first order) around $E(X)=x_{0}$, viz.,

$$
Y=\Delta(X) \approx \Delta\left(x_{0}\right)+\Delta^{\prime}\left(x_{0}\right)\left(X-x_{0}\right)+\varepsilon
$$

where $\varepsilon$ denotes the remainder term. Thus, to the first-order, the mean and variance of $Y=\Delta(X)$ are given by (B.7) and (B.8), respectively:

$$
\begin{gathered}
E(Y)=E(\Delta(X))=\Delta\left(x_{0}\right) \\
\begin{aligned}
V(Y)=V(\Delta(X)) & =E\left(\left[\Delta(X)-\Delta\left(x_{0}\right)\right]^{2}\right) \\
& =E\left(\left[\Delta^{\prime}\left(x_{0}\right)\left(X-x_{0}\right)\right]^{2}\right) \\
& =\left(\Delta^{\prime}\left(x_{0}\right)\right)^{2} E\left(\left(X-x_{0}\right)^{2}\right) \\
& =\left(\Delta^{\prime}\left(x_{0}\right)\right)^{2} V(X)
\end{aligned}
\end{gathered}
$$

From (B.8), it is evident that the expectation of $E(\Delta(X))$ given by (B.7) achieves a very good accuracy if $\Delta(X)$ is nearly a flat function with respect to its argument $X$. This observation, in turn can assist us in explaining why different Dirac delta approximations (based on different approximations for the $Q$-function) tend to perform better than the others for a specified modulation scheme and the fading environment. 
International Journal of Computer Networks \& Communications (IJCNC) Vol.5, No.1, January 2013

\section{REFERENCES}

[1] M. K. Simon and M.S. Alouini, Digital Communications over Fading Channels, New York: Wiley, $2^{\text {nd }}$ Edition, 2005.

[2] J. G. Proakis and M. Salehi, Digital Communications, McGraw Hill, Fifth Edition, 2007.

[3] P. O. Borjesson and C. E. Sundberg, "Simple Approximations of the Error Function $Q(x)$ for Communications Applications,“IEEE Trans. Communications, vol. 27, no. 3, pp. 639-643, 1979.

[4] M. Chiani, D. Dardari, and M. K. Simon, "New Exponential Bounds and Approximations for the Computation of Error Probability in Fading Channels," IEEE Trans. Wireless Communications, vol. 2, no. 4, pp. 840-845, July 2003.

[5] G. K. Karagiannidis and A. S. Lioumpas, "An Improved Approximation for the Gaussian $Q$ Function,“ IEEE Communications Letters, vol. 11, no. 8, pp. 644-646, Aug. 2007.

[6] Y. Isukapalli and B. D. Rao, "An Analytically Tractable Approximation for Gaussian $Q$-Function," IEEE Communication Letters, vol. 12, no. 9, pp. 669-671, Sept. 2008.

[7] Y. Chen and N. C. Beaulieu, "A Simple Polynomial Approximation to the Gaussian $Q$-function and Its Application,” IEEE Communication Letters, vol. 13, pp. 124-126, Feb. 2009.

[8] P. Loskot and N. C. Beaulieu, "Prony and Polynomial Approximations for Evaluation of the Average Probability of Error over Slow-Fading Channels," IEEE Trans. Vehicular Technology, pp. 1269-1280, March 2009.

[9] W. M. Jang, “A Simple Upper Bound of the Gaussian $Q$-Function with Closed-Form Error Bound," IEEE Communications Letters, vol. 15, no. 2, pp. 157-159, Feb. 2011.

[10] M. Lopez-Benitez and F. Casadevall, "Versatile, Accurate, and Analytically Tractable Approximation for the Gaussian Q-Function," IEEE Trans. Communications, vol. 59, pp. 917-922, April 2011.

[11] Q. Shi and Y. Karasawa, "An Accurate and Efficient Approximation to the Gaussian $Q$-Function and its Applications in Performance Analysis in Nakagami-m Fading," IEEE Communications Letters, vol. 15, pp. 479-481, May 2011.

[12] S. Chang, P. Cosman and L. Milstein, "Chernoff-Type Bounds for the Gaussian Error Function," IEEE Trans. Communications, vol. 59, pp. 2939-2944, Nov. 2011.

[13] O. Olabiyi, and A. Annamalai, "ASER Analysis of Cooperative Non-Regenerative Relay Systems over Generalized Fading Channels,” Proc. IEEE ICCCN’11, Maui, Aug. 2011.

[14] O. Olabiyi and A. Annamalai, "Invertible Exponential-Type Approximations for the Gaussian Probability Intergral $Q(x)$ with Applications," IEEE Wireless Communication Letters, vol. 1, no. 5, pp. 544-547, Oct. 2012.

[15] W. M. Jang, "Quantifying Performance in Fading Channels Using the Sampling Property of a Delta Function," IEEE Communications Letters, vol. 15, no. 3, pp. 266-268, March 2011.

[16] W. M. Jang, "Quantifying Performance of Cooperative Diversity using the Sampling Property of a Delta Function,” IEEE Trans. Wireless Communications, vol. 10, no. 7, pp. 2034-2039, July 2011.

[17] H. S. Abdel-Ghaffar and S. Pasupathy, "Asymptotical Performance of $M$-ary and Binary Signals over Multipath/Multichannel Rayleigh and Rician Fading," IEEE Trans. Communications, vol. 43, no. 11, pp. 2721-2731, Nov. 1995.

[18] Z. Wang and G. B. Giannakis, "A Simple and General Parameterization Quantifying Performance in Fading Channels,"IEEE Trans. Communications, vol. 51, no. 8, pp. 1389-1398, Aug. 2003.

[19] A. Nasri, R. Schober and Y. Ma, "Unified Asymptotic Analysis of Linearly Modulated Signals in Fading, Non-Gaussian Noise, and Interference," IEEE Trans. Communications, vol. 56, no. 6, pp. 980-990, June 2008.

[20] http://en.wikipedia.org/wiki/Multinomial theorem.

[21] A. Annamalai, C. Tellambura and V. K. Bhargava, "A General Method for Calculating Error Probabilities over Fading Channels," IEEE Trans. Communications, vol. 53, no. 5, pp. 841-852, May 2005. 
International Journal of Computer Networks \& Communications (IJCNC) Vol.5, No.1, January 2013

[22] E. Adebola, O. Olabiyi and A. Annamalai, "On the Dirac Delta Approximation and the MGF Method for ASER Analyses of Digital Communications over Fading Channels," to appear in the IEEE Communications Letters, 2013.

[23] A. Laourin, M. S. Alouini, S. Affes, A. Stephenne, "On the Performance Analysis of Composite Multipath/Shadowing Channels Using the G-distribution," IEEE Trans. Communications, vol. 57, no. 4, pp. 1162-1170, April 2009..

[24] N. Y. Ermolova, "Moment Generating Functions of the Generalized $\eta-\mu$ and $\kappa-\mu$ Distributions and Their Applications to Performance Evaluations of Communication Systems," IEEE Communications Letters, vol. 12, no. 7, pp. 502-504, July 2008.

\title{
ACKNOWLEDGEMENTS
}

This work is supported in part by funding from the Air Force Research Laboratory/Clarkson Aerospace and the National Science Foundation (0931679 \& 1040207).

\begin{abstract}
Authors
Dr. Annamalai is presently the Director of Center of Excellence for Communication Systems Technology Research, a Texas A\&M Board of Regents approved University Research Center at the Prairie View A\&M University (PVAMU), and a tenured faculty member in the Department of Electrical and Computer Engineering. He has over 18 years of research and teaching experience in wireless communications at Motorola, University of Victoria, Air Force Research Laboratory, Virginia Tech and PVAMU with over 200 peer-reviewed publications and 5 book chapters. Dr. Annamalai has been honored by his

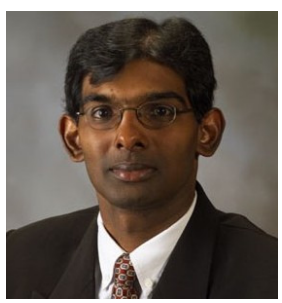
colleagues on numerous occasions for his excellence in research including winning the 2011 Roy G. Perry College of Engineering Outstanding Faculty (Research) Award, IEEE Leon Kirchmayer Prize Paper award, ASEE/AFOSR Summer Faculty Fellowships, NSERC Doctoral Prize, CAGS/UMI Distinguished Doctoral Dissertation Award, IEEE VTS/Motorola Daniel E. Noble Fellowship, among others. His current research interests include cooperative spectrum sensing, compressive sensing, cross-layer design for scalable multimedia transmission and cooperative wireless communications.
\end{abstract}

Eyidayo F. Adebola received the B.Sc. degree in Electronic \& Electrical Engineering from Obafemi Awolowo University, Ile-Ife, Nigeria and M.S. degree in Electrical Engineering from Prairie View A\&M University, Texas. He is currently working towards his $\mathrm{PhD}$. degree in Electrical Engineering at Prairie View A\&M University. He was the recipient of the 2012 Roy G. Perry College of Engineering Outstanding Masters Student of the Year Award. His broad research interests are in the field of wireless communications including cognitive radio networks and performance analysis and cross-layer optimization of cooperative wireless communications.

Oluwatobi O. Olabiyi received the B.Sc. degree in Electronic \& Electrical Engineering from Obafemi Awolowo University, Ile-Ife, and the M.S. degree in Electrical Engineering from Prairie View A\&M University, Texas. He has coauthored more than 25 publications presented at international conferences and published in highly referred journals. He was the recipient of the Roy G. Perry College of Engineering Outstanding Masters Student of the Year Award (2011) and the National Society of Black Engineer's Golden Torch Award for Graduate Student of Year (2012). He is presently a $\mathrm{PhD}$ candidate at Prairie View A\&M

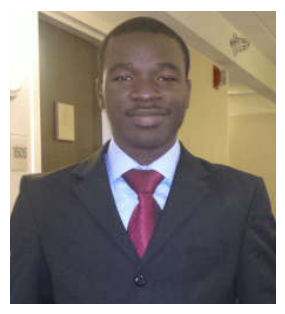

University. His research interests are in wireless communications including, dynamic spectrum access, MIMO and cooperative communications, statistical signal processing, compressive sensing, machine learning and optimization. 